\title{
FSH aggravates bone loss in ovariectomised rats with experimental periapical periodontitis
}

\author{
HUA QIAN, XIAOYUE GUAN and ZHUAN BIAN
}

\begin{abstract}
State Key Laboratory Breeding Base of Basic Science of Stomatology and Key Laboratory of Oral Biomedicine Ministry of Education, School and Hospital of Stomatology, Wuhan University, Wuhan, Hubei 430079, P.R. China
\end{abstract}

Received July 21, 2015; Accepted July 22, 2016

DOI: $10.3892 / \mathrm{mmr} .2016 .5613$

\begin{abstract}
Periapical bone loss is one of the prominent pathological and clinical features of periapical periodontitis. Previous studies have demonstrated that follicle-stimulating hormone (FSH) could directly affect skeletal remodelling by stimulating the formation and the function of osteoclasts in vitro and in vivo. However, the effect of FSH on periapical bone loss remained to be fully elucidated. In the current study, a rat model was established in order to verify the effect of FSH in experimental periapical lesions. It was identified that FSH aggravated the bone loss of periapical lesions. In addition, RANKL-, TRAP-, TNF- $\alpha$ - and IL- $1 \beta$-positive cells were increased significantly in FSH-treated groups, which indicated that the function of FSH in bone loss may be mediated through the increasing activity of osteoclasts and the increased secretion of inflammatory cytokines. The results of the current study suggested that FSH, independent of oestrogen, may aggravate periapical bone loss by FSH receptors, which may serve an important role in the immune and inflammatory response of the host to root canal and periradicular infection during menopause.
\end{abstract}

\section{Introduction}

Oestrogen deficiency has been suggested to be the predominant cause of post-menopausal osteoporosis (1). Accompanied by the withdrawal of sex steroids in mammals, serum levels of the pituitary hormone follicle-stimulating hormone (FSH) are markedly increased (2,3). FSH exhibits osteoclastogenic and proresorptive actions in a Gi2 $\alpha$-coupled FSH receptor (FSHR) identified in human and mouse osteoclasts and their

Correspondence to: Professor Zhuan Bian, State Key Laboratory Breeding Base of Basic Science of Stomatology and Key Laboratory of Oral Biomedicine Ministry of Education, School and Hospital of Stomatology, Wuhan University, 237 Luoyu Road, Wuhan, Hubei 430079, P.R. China

E-mail:bz@whuss.com

Key words: follicle-stimulating hormone, leuprorelin, periapical lesion, ovariectomy oestrogen-independent precursors (4). In osteoclasts, FSHR activation enhances the phosphorylation of downstream receptor activator of nuclear factor $\kappa \mathrm{B}$ ligand (RANKL)-sensitive kinases, extracellular signal-regulated kinases, protein kinase $\mathrm{B}$ and inhibitor of $\kappa \mathrm{B}$ to transduce the proresorptive actions of RANKL (5). The highly circulating FSH exhibits a strong positive correlation with bone remodelling markers; alterations in FSH circulating levels can predict bone mass remodeling (6). A recent study additionally observed that FSH-mediated effects on the secretion of inflammatory factors, particularly interleukin-1 (IL-1) and tumour necrosis factor $\alpha$ (TNF- $\alpha$ ), may be partly involved in regulating the resorptive activity of osteoclasts (7).

Periapical bone loss is a prominent pathological and clinical feature of periapical periodontitis, which begins as a bacterial infection in the dental pulp; inflammatory cells are then recruited and cytokines are subsequently generated (8). It has been identified that the regulatory mechanisms of apical periodontitis are complex, and remain to be fully elucidated. Bone-resorptive cytokines, including IL-1 and TNF- $\alpha$, and bone resorptive factors, in particular RANKL, serve key roles in pulpal and periapical pathogenesis, including bone destruction (9). In addition, systemic factors, such as hormones, are able to influence periapical pathogenesis. In the periapical lesions of ovariectomised (OVX) rats, more severe bone destruction has been observed (10). Increased RANKL expression levels have been observed in periapical lesions of OVX rats, which are attributed to oestrogen deficiency (11). Further studies have indicated that OVX mice yield high FSH levels as oestrogen levels are reduced. FSH, independent of oestrogen, can aggravate alveolar bone loss through FSHR in periodontitis; the FSH inhibitor leuprorelin (LE) elicits protective effects on alveolar bone loss resulting from experimental periapical lesions of OVX rats (12). However, it remains unclear whether FSH serves a direct role in the progression of apical periodontitis.

The current study aimed to assess the effects and the associated mechanisms of FSH on apical periodontitis-associated bone loss. Enzyme histochemical examination, radioimmunoassay and immunohistochemical staining were conducted in the present study in order to detect the following: FSHR, a novel positive biomarker of osteoclasts; RANKL, a positive regulator of osteoclastogenesis and osteoclastic activity; osteoprotegerin (OPG), a negative regulator of osteoclastogenesis 
A

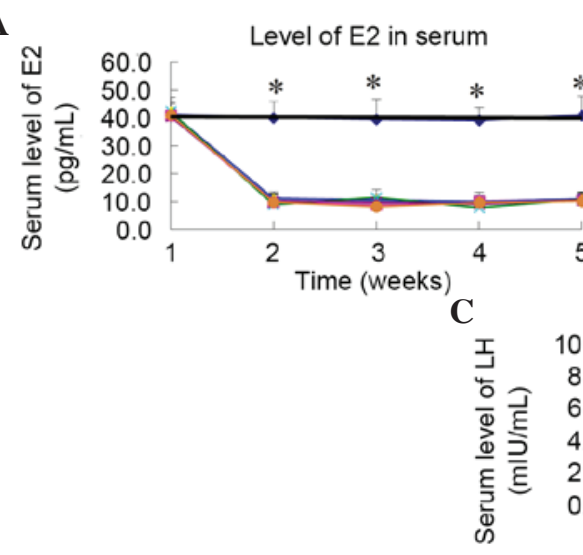



B

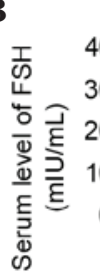

Level of $\mathrm{FSH}$ in serum



Figure 1. Levels of E2, FSH and LH in serum. (A) From 7 days after ovariectomy, the levels of E2 were significantly reduced in the serum of all OVX groups vs. Sham group, and plateaued at a level $\sim 1 / 4$ of that of the Sham group $(\mathrm{P}<0.01)$, with no statistical differences among the OVX groups $(\mathrm{P}>0.05)$. (B and $\mathrm{C})$ Levels of FSH and LH in serum were significantly increased vs. the Sham group after ovariectomy, and this increase was prevented by application of LE $(\mathrm{P}<0.05)$. Levels of FSH rapidly increased following application in the FSH-treated group, with levels approximately $10 x$ greater than that of the OVX group (P<0.05). Levels of $\mathrm{LH}$ in the OVX $+\mathrm{LE}+\mathrm{LH}$ group rapidly increased and plateaued from the third week after administration of $\mathrm{LH}(\mathrm{P}<0.05)$. ${ }^{\ominus} \mathrm{P}<0.05$ vs. the $\mathrm{OVX}$-only and FSH treatment groups; ${ }^{\#} \mathrm{P}<0.05$ vs. the non-FSH treatment group; ${ }^{\Delta} \mathrm{P}<0.05$ vs. the non-LH treatment groups. E2, estradiol; FSH, follicle-stimulating hormone; LH, luteinizing hormone; OVX, ovariectomised; LE, leuprorelin.

and osteoclastic activity; and TNF- $\alpha$ and IL-1 $\beta$, inflammatory cytokines.

\section{Materials and methods}

Animal experimental groups. A total of 90 12-week-old female Sprague-Dawley rats, weighing 180-200 g, were purchased from the Laboratory Animal Centre of Wuhan University (Wuhan, China) and were housed with a temperature of $24-26^{\circ} \mathrm{C}$ and a $12 \mathrm{~h}$ light/dark cycle. The rats were randomly assigned into the following six groups with 15 rats/group: i) Group 1, Sham surgery $+0.1 \mathrm{~mol} / 1$ phosphate-buffered saline (PBS; pH 7.4) as a vehicle, ii) group 2, bilateral OVX + vehicle, iii) group 3, bilateral OVX + $1.6 \mathrm{mg} / \mathrm{kg} \mathrm{LE}$ (Takeda Pharmaceutical Company, Ltd., Osaka, Japan), iv) group 4, bilateral $\mathrm{OVX}+1.6 \mathrm{mg} / \mathrm{kg} \mathrm{LE}+1.85 \mu \mathrm{g} / \mathrm{kg}$ luteinizing hormone $(\mathrm{LH}$ Serono; Merck Millipore, Geneva, Switzerland), v) group 5, bilateral OVX + $1.6 \mathrm{mg} / \mathrm{kg} \mathrm{LE}+3 \mu \mathrm{g} / \mathrm{kg}$ FSH (Serono; Merck Millipore) and vi) group 6 , bilateral OVX $+3 \mu \mathrm{g} / \mathrm{kg}$ FSH. The experimental protocols were approved by the Institutional Animal Care and Use Committee of Wuhan University.

Ovariectomy for rats. Rats were anaesthetised by intraperitoneal injection $3 \mathrm{mg} / \mathrm{ml}$ pentobarbital $(10 \mathrm{ml} / \mathrm{kg})$ (Veterinary Institute of Military Supplies University, Changchun, China). Subsequently, the rats in groups 2-6 were subjected to bilateral OVX. The rats in group 1 were subjected to Sham surgeries with similar-sized fatty tissues near to the removed ovaries. Subsequent to surgery, LE, Vehicle, LH or FSH were subcutaneously injected into the rats in groups 3-5, groups 1 and 2, group 4 or groups 5 and 6 at the relevant concentrations, respectively. As a slow-release formulation, LE can maintain effective blood concentration and inhibit increases of circulating LH and FSH levels for a minimum of 4 weeks subsequent to injection.

Induction of experimental periapical lesions. At the 7th day following surgery, all rats were anaesthetised as previously described $(13,14)$. The pulps of the mandibular first molars were exposed using a \#1/2 round bur under a surgical microscope (M320 F12, Leica Microsystems GmbH, Wetzlar, Germany). The exposure size was equal to the diameter of the bur to avoid furcal perforation. The exposed pulp chambers were left to the oral environment without any restoration during the entire experimental period. After pulpal exposure, FSH, LH, LE and vehicle were subcutaneously injected in the back of rats daily.

Measurement of estradiol (E2), FSH and LH serum levels. On the 7th, 14th and 21st day after pulpal exposure, the venous blood samples were collected and centrifuged at $1,000 \mathrm{x} \mathrm{g}$ for $10 \mathrm{~min}$ at $4^{\circ} \mathrm{C}$. The serum was then separated and acquired to test E2, FSH and LH levels using a radioimmunoassay kit (North institute of Biological Technology, Beijing, China) according to the manufacturer's instructions.

Histological observation. On the 7th, 14th and 21st day after pulpal exposure, rats were anaesthetised with an intraperitoneal injection of $3 \mathrm{mg} / \mathrm{ml}$ pentobarbital $(10 \mathrm{ml} / \mathrm{kg}$; Veterinary Institute of Military Supplies University, Changchun, China) and sacrificed by cervical dislocation. Bilateral mandibles were removed and fixed with $4 \%$ paraformaldehyde at $4^{\circ} \mathrm{C}$ for 2 days. In order for histological observation to be conducted, samples were decalcified with $10 \%$ ethylenediaminetetraacetic acid (EDTA) at $4^{\circ} \mathrm{C}$ for $\sim 6$ weeks. The decalcified specimens were then embedded in paraffin and cut into pieces at a thickness of $4 \mu \mathrm{m}$ using a microtome from Jung AG (Heidelberg, Germany). The sections were then stained with haematoxylin/eosin or immunohistochemistry antibodies. Subsequent to haematoxylin/eosin staining, samples were photographed under a light microscope. The areas of periapical lesions were measured by SPOT RT software, version 3.5 (Diagnostic Instruments, Inc., Sterling Heights, MI, USA) in three random HE-stained sections in a double-blind manner as previously described $(10,15)$.

Immunohistochemistry. Subsequent dewaxing and rehydration, the slides were treated with $3 \%$ hydrogen peroxide at 



Figure 2. Histological analysis of periapical lesions in each group. (A) Sham, (B) OVX, (C) OVX + FSH, (D) OVX + LE, (E) OVX + LE + FSH and (F) OVX + LE + LH groups. (G) Quantitative analysis of periapical lesions in each group. Compared with those in the Sham group, specimens in the OVX groups exhibited significant increases in bone loss of periapical lesions $(\mathrm{P}<0.05 ; \mathrm{A}, \mathrm{B}$ and $\mathrm{G})$, which were reversed by the administration of $\mathrm{LE}$ or $\mathrm{LE}+\mathrm{LH}$ ( $\mathrm{P}>0.05 ; \mathrm{D}-\mathrm{G})$. Bone loss of periapical lesions was significantly increased vs. those in the Sham and OVX groups after administration of FSH (P<0.05; C, E and G). Data is presented as the mean \pm standard deviation. " $\mathrm{P}<0.05$ vs. the OVX only group and the FSH treatment group; $\mathrm{P}<0.05$ vs. non-FSH treatment groups. OVX, ovariectomised; FSH, follicle-stimulating hormone; LE, leuprorelin; LH, luteinizing hormone.

$37^{\circ} \mathrm{C}$ for 15 min to inhibit any endogenous peroxidase activity. The slices were then incubated separately with the following primary antibodies: Polyclonal goat anti-FSHR (1:200; Santa Cruz Biotechnology, Inc., Dallas, TX, USA; cat. no. sc-7797), polyclonal goat anti-RANKL (1:100; Santa Cruz Biotechnology, Inc.; cat. no. sc-7628), polyclonal goat anti-OPG (1:200; Santa Cruz Biotechnology, Inc.; cat. no. sc-8468), polyclonal rabbit anti-TNF- $\alpha$ (1:300; Bioss, Beijing, China; cat. no. 2081R), monoclonal hamster anti-IL-1 $\beta$ (1:300; Biolegend, Inc., San Diego, CA, USA; cat. no. 503501) for 24 h at $4^{\circ} \mathrm{C}$. Subsequent to incubation, the primary antibody was removed by washing the slides thrice with PBS for $5 \mathrm{~min}$. The sections were incubated with secondary antibody kits from ZSGB-Bio
(Beijing, China) according to the manufacturer's protocols. The kits were as follows: Polink-2 Plus ${ }^{\circledR}$ Polymer HRP Detection system for the antibodies raised in goat; and SPlink Detection kit (Biotin-Streptavidin HRP Detection system) for the remaining primary antibodies. The slides were stained for approximately $30-40 \mathrm{sec}$ with diaminobenzidine and the nucleus was counterstained with haematoxylin for $10 \mathrm{sec}$.

Enzyme histochemical examination. Periapical region slices were used to detect TRAP activity using the TRAP Detection kit (Sigma-Aldrich; Merck Millipore, Darmstadt, Germany). In brief, EDTA-decalcified tissue sections were rehydrated and incubated for $1 \mathrm{~h}$ at $37^{\circ} \mathrm{C}$ with the TRAP staining solution. 

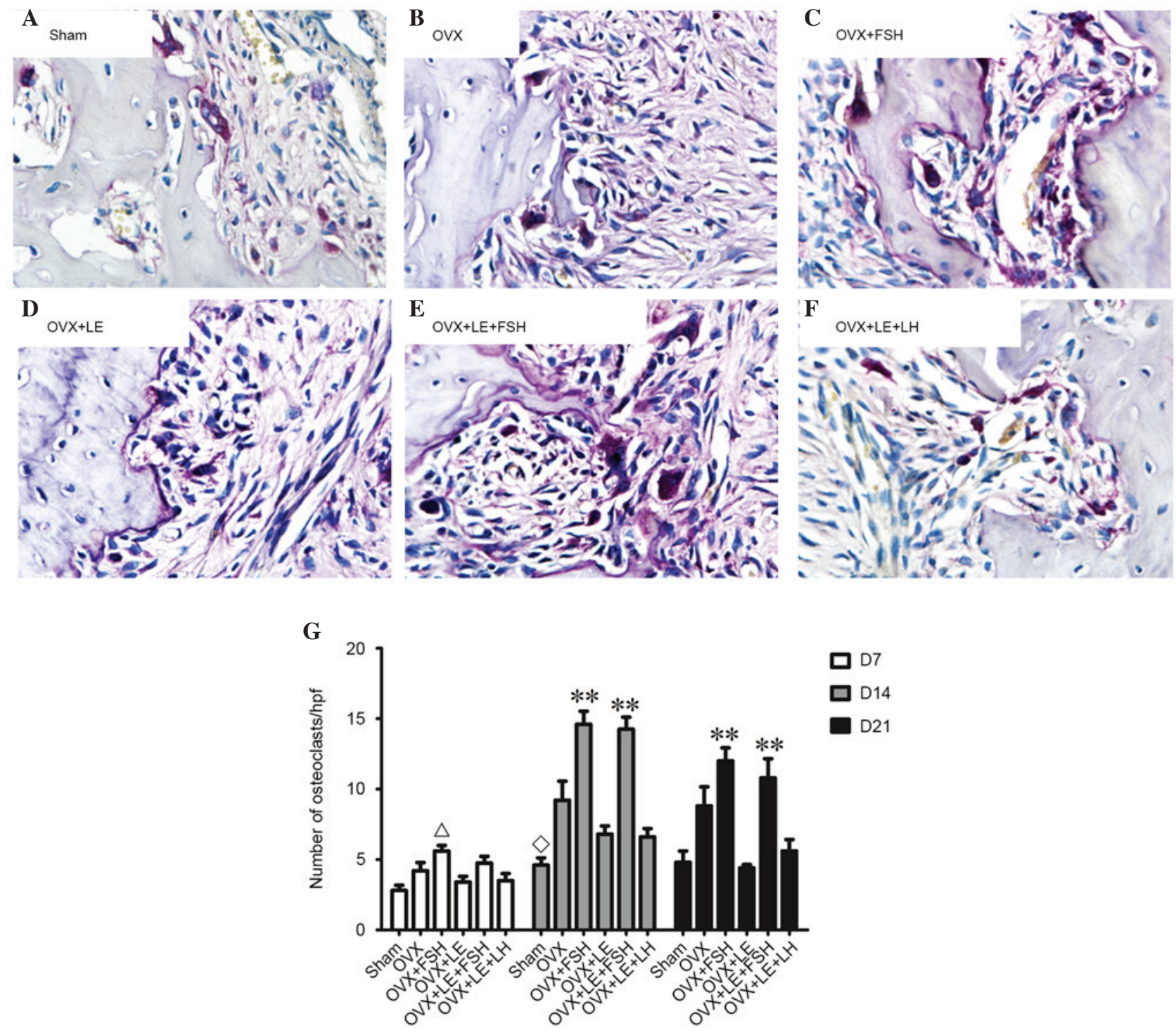

Figure 3. Immunohistochemical observation of osteoclasts. (A) Sham, (B) OVX, (C) OVX + FSH, (D) OVX + LE, (E) OVX + LE + FSH and (F) OVX + LE + LH groups. Tartrate-resistant acid phosphatase-positive cells were stained dark red to purple, and multinuclear cells were considered osteoclasts. (G) Quantitative analysis of osteoclast number in each group. On the 7th, 14th and 21st day after pulpal exposure, the numbers of osteoclasts in OVX group were increased compared with that of the Sham group $(\mathrm{P}<0.01 ; \mathrm{A}, \mathrm{B}$ and $\mathrm{G})$. The numbers of osteoclasts in the OVX $+\mathrm{LE}+\mathrm{LH}$ and $\mathrm{OVX}+\mathrm{LE}$ groups were significantly greater than those in the OVX groups $(\mathrm{P}<0.01, \mathrm{C}, \mathrm{D}$ and $\mathrm{G})$, with no significant differences observed between them. The numbers of osteoclasts were significantly greater in the OVX + FSH and OVX + LE + FSH groups compared with those in the OVX and OVX + LE groups $(\mathrm{P}<0.01, \mathrm{E}, \mathrm{F}$ and G). Data is presented as the mean \pm standard deviation. ${ }^{\mathrm{A}} \mathrm{P}<0.05$ vs. the sham $\mathrm{D} 7$ group; ${ }^{\circ} \mathrm{P}<0.05$ vs. the OVX D14 group and the FSH treatment group; ${ }^{* *} \mathrm{P}<0.01 \mathrm{vs}$. non-FSH treatment groups on D14 and D21. OVX, ovariectomised; FSH, follicle-stimulating hormone; LE, leuprorelin; LH, luteinizing hormone.

Following completion of incubation, these sections were stained with haematoxylin. Certain sections were incubated in a substrate-free medium, which were used as controls for TRAP activity.

Statistical analysis. For each specimen, positive cells from five randomly selected areas at the periapical region were counted at a magnification of $\mathrm{x} 400$ under a light microscope. GraphPad Prism software, version 5.0 for Windows (GraphPad Software, Inc., La Jolla, CA, USA) was used to analyse the data. The differences in lesion size and the average number per high-power field were analysed by one-way analysis of variance and post-hoc Tukey or Bonferroni multiple comparison tests were conducted between the subgroups.

\section{Results}

Levels of E2, FSH and LH in serum. From 7 days after ovariectomy, compared with the Sham group, levels of E2 in the serum of all OVX groups were reduced significantly and plateaued at a level approximately $1 / 4$ of that of the Sham group $(\mathrm{P}<0.01)$, with no statistical differences identified among the OVX groups ( $\mathrm{P}>0.05$; Fig. 1A). The levels of FSH and LH observed in the serum were increased significantly, when compared with that of the Sham group subsequent to ovariectomy, however, this increase was prevented by the application of LE $(\mathrm{P}<0.05$; Fig. 1B and C). The levels of FSH were increased markedly subsequent to application of FSH in the FSH-treated group, with the levels observed approximately 10x greater than that observed in the OVX group $(\mathrm{P}<0.05$; 

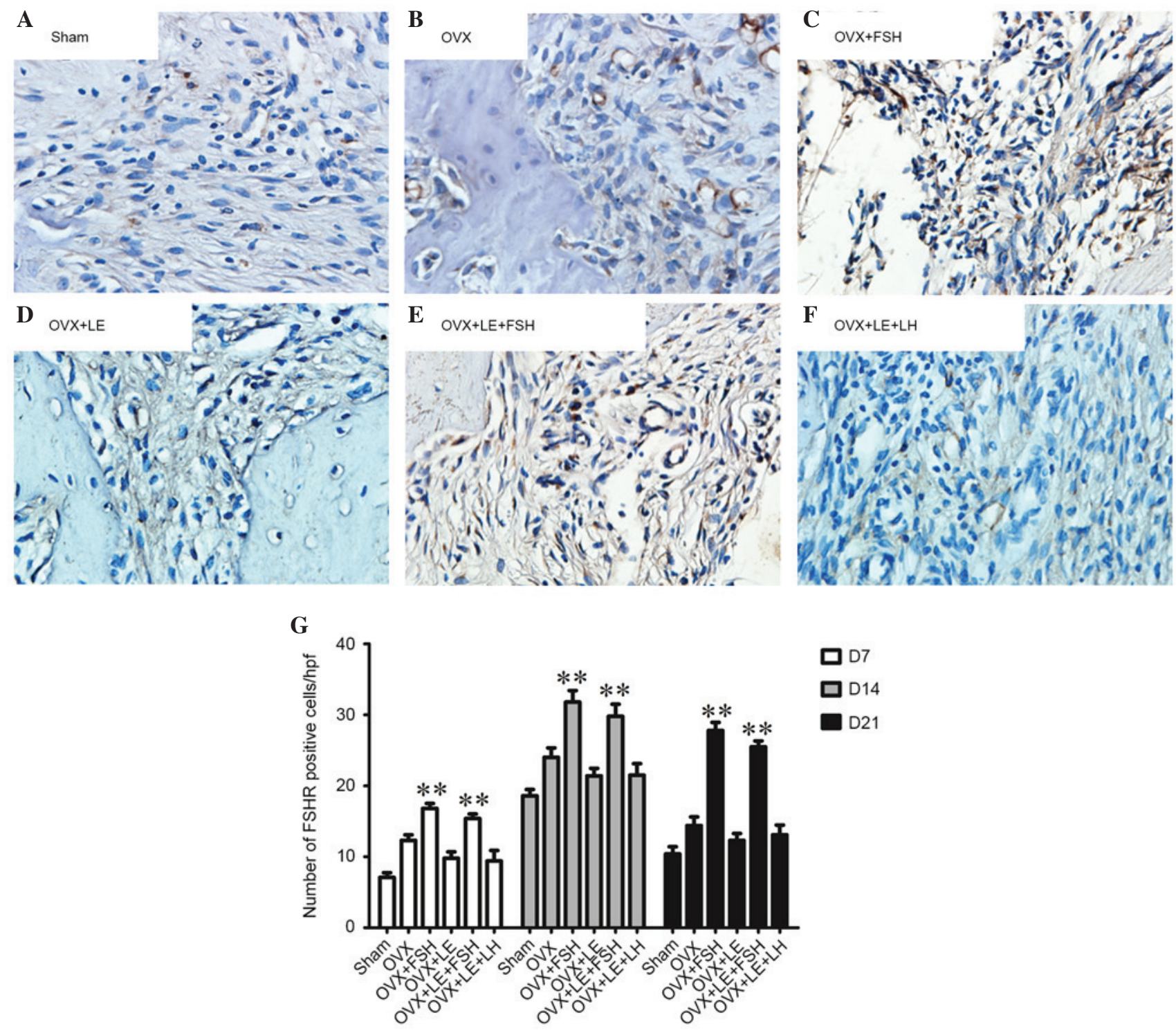

Figure 4. Immunohistochemical observation of FSHR expression. (A) Sham, (B) OVX, (C) OVX + FSH, (D) OVX + LE, (E) OVX + LE + FSH and (F) OVX + LE + LH groups. (G) Quantitative analysis of FSHR expression in each group. FSHR-positive cells were stained brown. On the 7th, 14th and 21st day after pulpal exposure, the numbers of FSHR-positive cells in OVX group were significantly greater than that of the Sham group (P<0.05; A, B and G). In the FSH-treated groups, the number of FSHR-positive cells was increased significantly compared with those in the Sham, OVX or OVX + LE groups $(\mathrm{P}<0.05 ; \mathrm{C}-\mathrm{E}$ and G). There was no difference between the OVX + LE and OVX + LE + LH groups ( $>>0.05 ; \mathrm{D}, \mathrm{F}$ and G). Data is presented as the mean \pm standard deviation. ${ }^{* *} \mathrm{P}<0.01$ vs. non-FSH treatment groups on D7, D14 and D21. FSH, follicle-stimulating hormone; FSHR, FSH receptor; OVX, ovariectomised; LE, leuprorelin; LH, luteinizing hormone.

Fig. 1B). The levels of $\mathrm{LH}$ measured in the OVX $+\mathrm{LE}+\mathrm{LH}$ group were increased rapidly and plateaued from the third week subsequent to administration of $\mathrm{LH}(\mathrm{P}<0.05$; Fig. 1C).

Histological analysis. The histological analysis is presented in Fig. 2. Compared with those in the Sham group, specimens in the OVX groups exhibited significant increases in bone loss of periapical lesions ( $\mathrm{P}<0.05$; Fig. $2 \mathrm{~A}, \mathrm{~B}$ and $\mathrm{G})$, which were reversed by administration of $\mathrm{LE}$ or $\mathrm{LE}+\mathrm{LH}(\mathrm{P}>0.05$; Fig. 2D-G). Compared with those in the Sham and OVX groups, bone loss of periapical lesions were significantly increased following administration of FSH ( $\mathrm{P}<0.05 ;$ Fig. $2 \mathrm{C}, \mathrm{E}$ and $\mathrm{G})$.

Immunohistochemical observation of osteoclasts and FSHR expression. TRAP-positive cells were stained dark red to purple, and multinuclear cells were considered osteoclasts (Fig. 3A-F). On the 7th, 14th and 21st day following pulpal exposure, the numbers of osteoclasts in the OVX group were higher than that of Sham group ( $\mathrm{P}<0.01$; Fig. $3 \mathrm{~A}, \mathrm{~B}$ and $\mathrm{G})$. The numbers of osteoclasts in OVX + LE + LH and the OVX + LE groups were significantly lower than those in the OVX group, with no significant differences between the $\mathrm{OVX}+\mathrm{LE}+\mathrm{LH}$ and OVX + LE groups themselves $(\mathrm{P}<0.01$; Fig. 3C, D and G). The numbers of osteoclasts were significantly greater in the OVX + FSH and OVX + LE + FSH groups than those in OVX and OVX + LE groups $(\mathrm{P}<0.01$; Fig. 3E, F and G).

FSHR-positive cells were stained brown (Fig. 4A-F). On the 7th, 14th and 21st day subsequent to pulpal exposure, the numbers of FSHR-positive cells in the OVX group were 

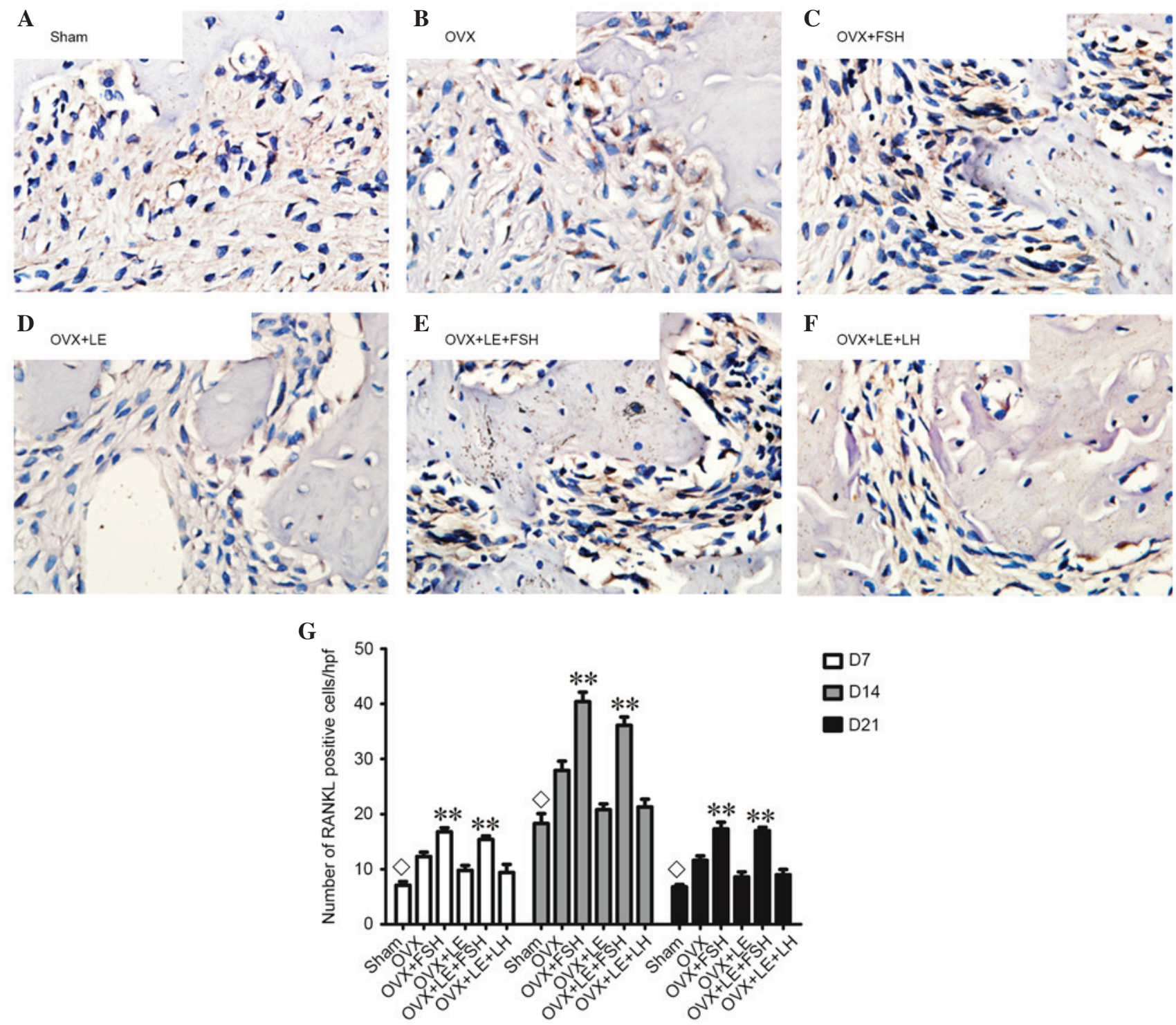

Figure 5. Immunohistochemical observation of RANKL expression. (A) Sham, (B) OVX, (C) OVX + FSH, (D) OVX + LE, (E) OVX + LE + FSH and (F) OVX + LE + LH groups. (G) Quantitative analysis of RANKL-positive expression in each group. RANKL-positive cells were stained brown. On the 7th, 14th and 21st day after pulpal exposure, the numbers of RANKL-positive cells in each group respectively reached the maximum value at 14 th day (P<0.05; G). At each time point, the number of RANKL-positive cells in FSH-treated groups was significantly greater compared with those in the corresponding non-FSH treatment groups $(\mathrm{P}<0.05$; B-E). The numbers of RANKL-positive cells were not observed to be significantly different between the OVX + LE and $\mathrm{OVX}+\mathrm{LE}+\mathrm{LH}$ groups at each time point $(\mathrm{P}>0.05 ; \mathrm{F}$ and $\mathrm{G})$. Data is presented as the mean \pm standard deviation. ${ }^{\circ} \mathrm{P}<0.05$ vs. the $\mathrm{OVX}$-only groups and the FSH treatment groups on D7, D14 and D21; ${ }^{* *} \mathrm{P}<0.01$ vs. non-FSH treatment groups on D7, D14 and D21. RANKL, receptor activator of nuclear factor $\kappa \mathrm{B}$ ligand; OVX, ovariectomised; FSH, follicle-stimulating hormone; LE, leuprorelin; LH, luteinizing hormone.

observed to be greater than that of the Sham group $(\mathrm{P}<0.05$; Fig. 4A, B and G). In the FSH-treated groups, the number of FSHR-positive cells was significantly increased compared with those in the Sham, OVX or OVX + LE groups $(\mathrm{P}<0.05$; Fig. 4C-E and G). There was no difference between the OVX + LE and OVX + LE + LH groups $(\mathrm{P}>0.05$; Fig. 4D, $\mathrm{F}$ and $\mathrm{G})$.

Comparison of RANKL and OPG expressions. RANKL- and OPG-positive cells were stained brown (Figs. 5A-F and 6A-F). On the 7th, 14th and 21st days after pulpal exposure, the number of RANKL- and OPG-positive cells in each group reached maximal values on day $14(\mathrm{P}<0.05$; Figs. $5 \mathrm{G}$ and $6 \mathrm{G})$. At each time point, the number of RANKL-positive cells in the FSH-treated groups was significantly greater than those in the corresponding non-FSH treatment groups $(\mathrm{P}<0.05$; Fig. 5B-E). There were no significant differences identified between the FSH-treated groups and the corresponding groups in the number of OPG-positive cells ( $>>0.05$; Fig. 6B-E). The numbers of RANKL- and OPG-positive cells were not significantly different between the OVX + LE and OVX + LE + LH groups at each time point ( $\mathrm{P}>0.05$; Figs. $5 \mathrm{~F}$ and $\mathrm{G}$ and $6 \mathrm{~F}$ and $\mathrm{G})$.

Comparison of TNF- $\alpha$ and IL-1 $\beta$ expressions. TNF- $\alpha-$ and IL-1 $\beta$-positive cells were stained brown and increased as the lesion expanded (Figs. 7A-F and 8A-F). The majority of these cells were likely inflammatory cells predominantly 

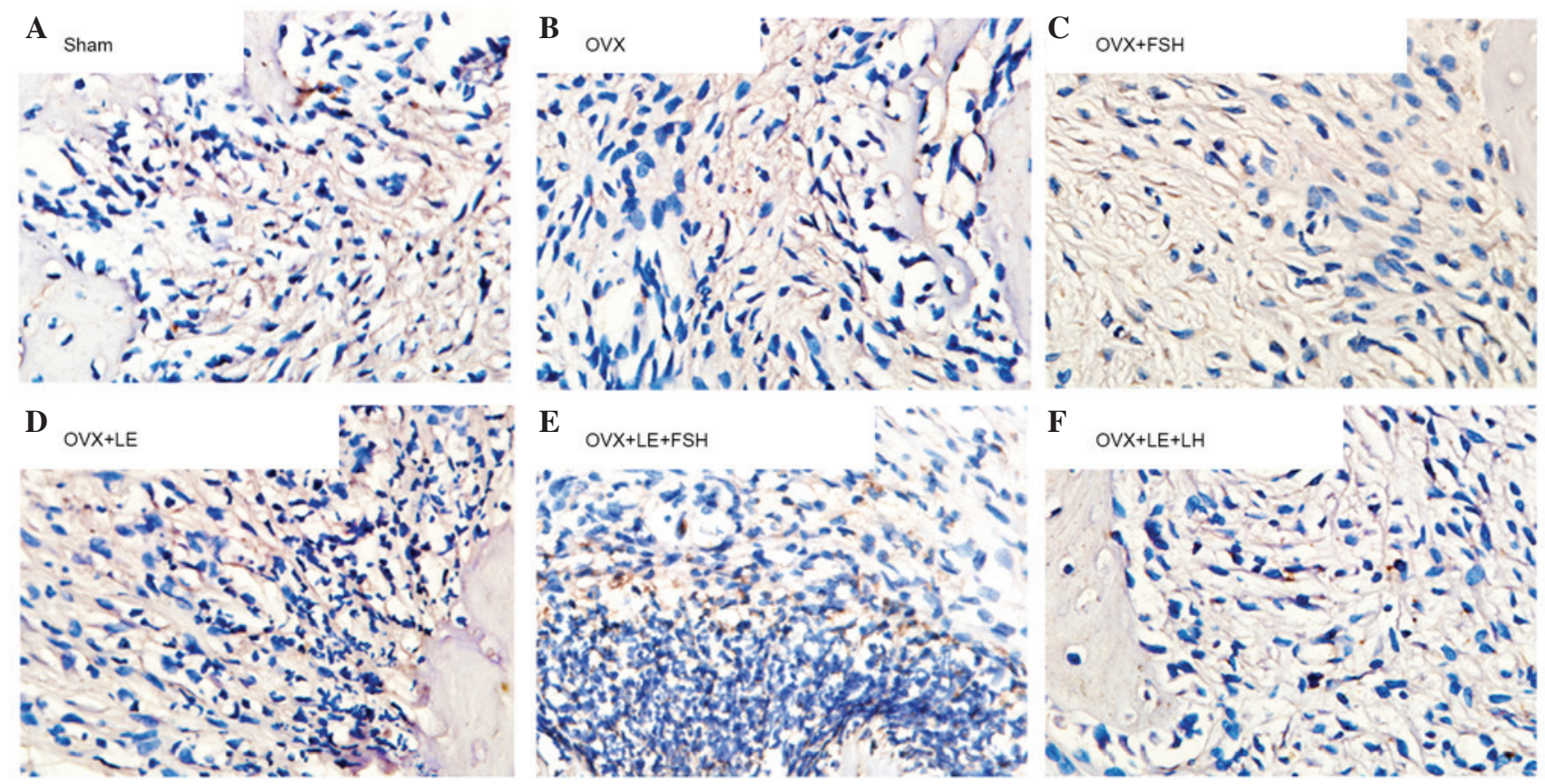

G



Figure 6. Immunohistochemical observation of OPG expression. (A) Sham, (B) OVX, (C) OVX + FSH, (D) OVX + LE, (E) OVX + LE + FSH and (F) OVX + LE + LH groups. (G) Quantitative analysis of OPG-positive expression in each group. OPG-positive cells were stained brown. On the 7th, 14th and 21 st day after pulpal exposure, the numbers of OPG-positive cells in each group peaked at day 14 (P<0.05; G). There were no significant differences observed between FSH-treated groups and corresponding groups in the number of OPG-positive cells (P>0.05; B-E). The numbers of OPG-positive cells exhibited no significant differences between OVX $+\mathrm{LE}$ and OVX $+\mathrm{LE}+\mathrm{LH}$ groups at each time point $(\mathrm{P}>0.05 ; \mathrm{F}$ and $\mathrm{G})$. Data is presented as the mean \pm standard deviation. ${ }^{\circ} \mathrm{P}<0.05$ vs. the OVX D14 group; ${ }^{\# \#} \mathrm{P}<0.01$ vs. the sham D7 group. OPG, osteoprotegerin; OVX, ovariectomised; FSH, follicle-stimulating hormone; LE, leuprorelin; LH, luteinizing hormone.

centralised at the periapical bone destruction areas and periodontal ligaments. The numbers of TNF- $\alpha$ - and IL-1 $\beta$-positive cells in the OVX group were increased compared with those in the Sham groups at each time point $(\mathrm{P}<0.05$; Figs. 7A and B and $8 \mathrm{~A}$ and $\mathrm{B}$ ). The numbers of TNF- $\alpha-$ and IL-1 $\beta$-positive cells were increased in the FSH-treated mice when compared with those in the corresponding non-FSH-treated mice ( $\mathrm{P}<0.05$; Figs. $7 \mathrm{~B}-\mathrm{E}$ and $8 \mathrm{~B}-\mathrm{E})$. The numbers of TNF- $\alpha$ - and IL-1 $\beta$-positive cells were not significantly different between the OVX + LE and OVX + LE + LH groups at each time point $(\mathrm{P}>0.05$; Figs. $7 \mathrm{~F}$ and $\mathrm{G}$ and $8 F$ and $G)$.

\section{Discussion}

Post-menopausal osteoporosis, a global public health problem, has been attributed to reducing oestrogen levels. FSH levels increase rapidly as oestrogen levels are reduced during the post-menopausal period (16). FSH directly affects skeletal remodelling by stimulating the formation and the function of osteoclasts in vitro and in vivo (16). In the current study, a rat model was established to verify the effects of FSH in experimental periapical lesions. The results indicated that FSH aggravated the bone loss of periapical lesions. In addition, RANKL-, TRAP-, TNF- $\alpha$ - and IL- $1 \beta$-positive cells 

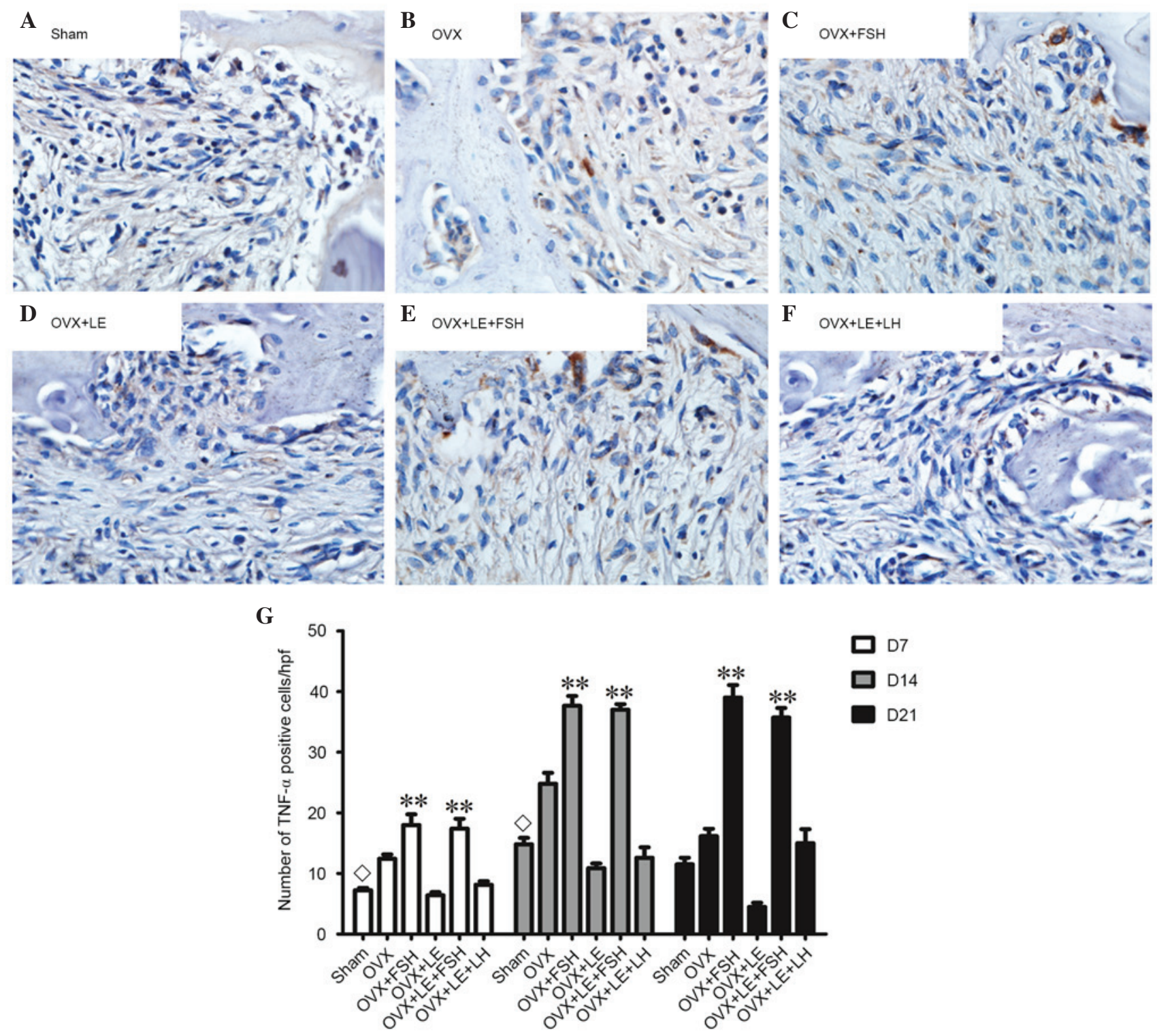

Figure 7. Immunohistochemical observation of TNF- $\alpha$ expression. (A) Sham, (B) OVX, (C) OVX + FSH, (D) OVX + LE, (E) OVX + LE + FSH and (F) OVX + LE + LH groups. (G) Quantitative analysis of TNF- $\alpha$-positive expression in each group. TNF- $\alpha$-positive cells were stained brown and increased as the lesion expanded. The numbers of TNF- $\alpha$-positive cells in the OVX group were larger than those in Sham groups at each time point $(\mathrm{P}<0.05 ; \mathrm{A}$ and $\mathrm{B})$. The numbers of TNF- $\alpha$-positive cells were larger in FSH-treated mice than those in the corresponding non-FSH-treated mice $(\mathrm{P}<0.05$; B-E). The numbers of TNF- $\alpha$-positive cells were not significantly different between the OVX + LE and OVX + LE + LH groups at each time point $(\mathrm{P}>0.05 ; \mathrm{F}$ and G). Data is presented as the mean \pm standard deviation. ${ }^{\circ} \mathrm{P}<0.05$ vs. the OVX-only group on $\mathrm{D} 7$ and $\mathrm{D} 14 ;{ }^{* * *} \mathrm{P}<0.01$ vs. the non-FSH treatment groups on D7, D14 and D21. TNF- $\alpha$, tumour necrosis factor $\alpha$; OVX, ovariectomised; FSH, follicle-stimulating hormone; LE, leuprorelin; LH, luteinizing hormone.

were increased significantly in the FSH-treated groups, which indicated that the function of FSH in bone loss may be mediated via the increasing activity of osteoclasts and the increased secretion of inflammatory cytokines.

Although ovariectomies markedly affect mineralisation density of mandibular and maxillary alveolar bones, ovariectomy alone cannot elucidate the effects of FSH (17). In the current study, a rat model was successfully established to verify the independent role of FSH by administering FSH, LH and LE based on OVX (12). In the current study, E2 levels did not differ among all the OVX groups. However, FSH levels significantly differed among the different OVX groups. For example, the comparison results of OVX vs. OVX + FSH group and OVX + LE vs. OVX $+\mathrm{LE}+\mathrm{FSH}$ group indicated the effects of extrinsic FSH in periapical bone loss.
Ovariectomy in mice has been reported to exacerbate induced periapical lesions; this condition is attributed to oestrogen deficiency $(10,18)$. In the present study, similar alterations in alveolar bone loss were observed in the OVX group compared with the Sham group. In addition, significant differences in the numbers of osteoclasts and the areas of bone loss were observed in the OVX group vs. OVX + FSH group and in OVX + LE group vs. OVX + LE + FSH group. The bone loss on day 21 in the OVX + FSH group was the most pronounced among all of the groups. Furthermore, the administration of $\mathrm{LE}$, an FSH inhibitor, resulted in a reduction of bone loss in the OVX + LE and OVX + LE + LH groups compared with the OVX group. No significant differences were identified between the OVX + LE and OVX + LE + LH groups. Therefore, it is suggested that FSH, independent of 

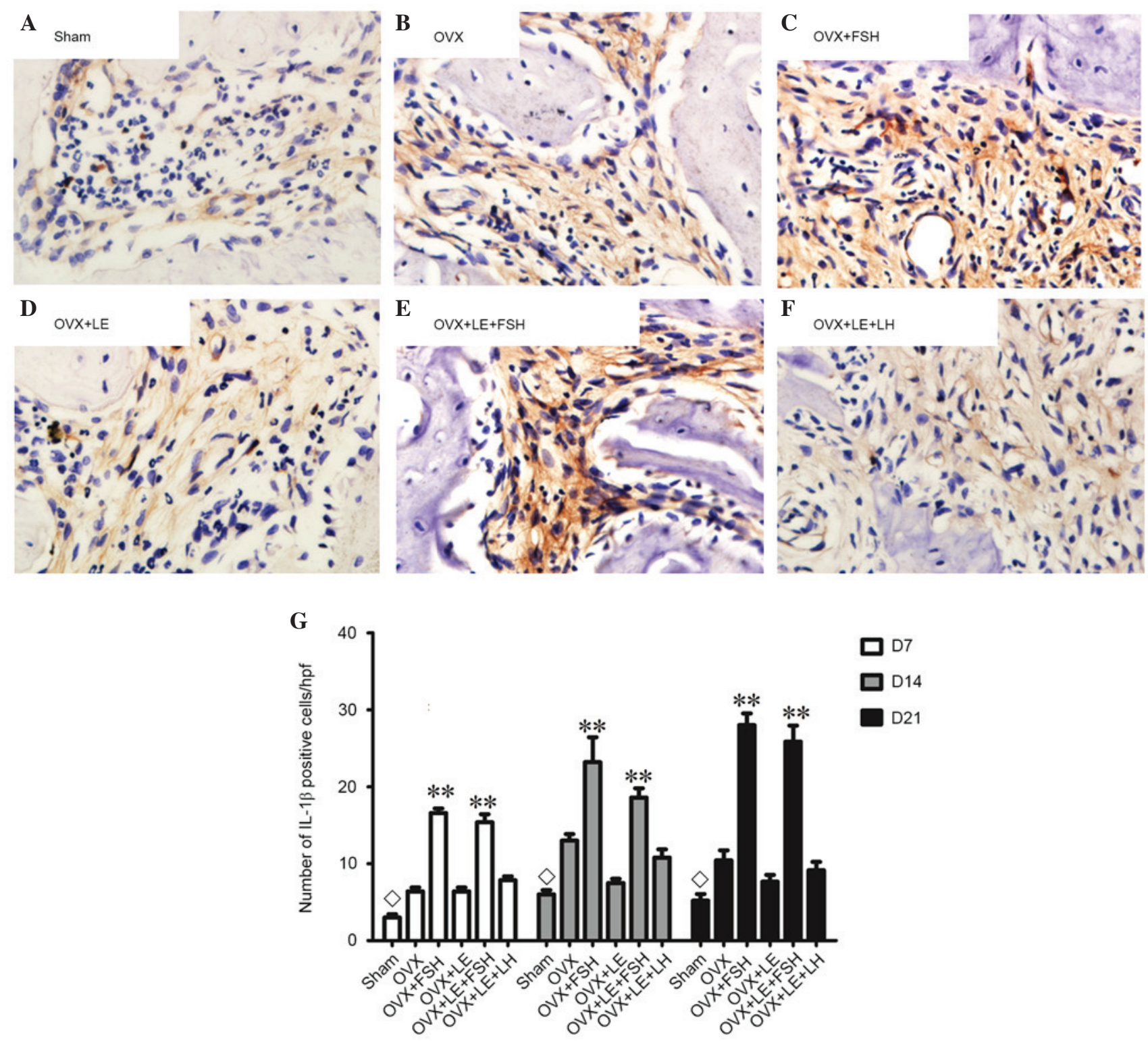

Figure 8. Immunohistochemical observation of IL-1 $\beta$ expression. (A) Sham, (B) OVX, (C) OVX + FSH, (D) OVX + LE, (E) OVX + LE + FSH and (F) OVX + LE + LH groups. (G) Quantitative analysis of IL-1 $\beta$-positive expression in each group. IL-1 $\beta$-positive cells were stained brown and increased as the lesion expanded. The numbers of IL-1 $1 \beta$-positive cells in OVX group were greater than those in the Sham groups at each time point $(\mathrm{P}<0.05 ; \mathrm{A}$ and $\mathrm{B})$. The numbers of IL-1 $\beta$-positive cells were greater in FSH-treated mice when compared with those in the corresponding non-FSH-treated mice $(\mathrm{P}<0.05$; $\mathrm{B}-\mathrm{E})$. The numbers of IL-1 $\beta$-positive cells were not observed to be significantly different between the OVX $+\mathrm{LE}$ and OVX $+\mathrm{LE}+\mathrm{LH}$ groups at each time point $(\mathrm{P}>0.05$; $\mathrm{F}$ and G). Data is presented as the mean \pm standard deviation. ${ }^{\circ} \mathrm{P}<0.05$ vs. the OVX-only group on D7, D14 and D21; ${ }^{* *} \mathrm{P}<0.01$ vs. the non-FSH treatment groups on D7, D14 and D21. IL-1 $\beta$, interleukin 1 $\beta$; OVX, ovariectomised; FSH, follicle-stimulating hormone; LE, leuprorelin; LH, luteinizing hormone.

oestrogen, can aggravate alveolar bone loss of induced periapical lesions.

In the current study, it was additionally identified that administration of FSH may significantly increase the expression of RANKL. The numbers of FSHR-positive cells were positively correlated with those of RANKL- and TRAP-positive cells. RANKL/OPG is an important factor influencing the production of osteoclasts $(19,20)$. FSH may also influence RANKL signalling via FSHR due to the fact that FSH and RANKL served an important role in osteoclastogenesis (6). RANKL expression in bone marrow cells of post-menopausal women has been reported to be greater than that of pre-menopausal women (21-23). Furthermore, RANKL is implicated in mediating increased bone resorption in early postmenopausal women (24). An additional study reported that RANKL-stimulated osteoclastogenesis is enhanced by FSH in human peripheral blood mononuclear cells (25). As an osteoblastic factor, OPG expression in the OVX + FSH group was unexpectedly greater than those in other groups at day 7 , which indicated that osteoblastogenesis was also accelerated in the early stages of high FSH level administration, as a type of reactive-protective response. In other groups, no significant differences in the expression of OPG were observed, which suggested that FSH may alter the balance of the RANKL/OPG system by increasing RANKL expression levels.

The results of the current study suggested that higher expression levels of TNF- $\alpha$ and IL- $1 \beta$ were present in the FSH-treated groups, when compared with those in the 
non-FSH-treated groups. This indicated that the effects of FSH on periapical lesions may be associated with the upregulation of TNF- $\alpha$ and IL-1 $\beta$ expression. Although oestrogen deficiency increases TNF- $\alpha$ expression in certain instances, such as after ovariectomy, FSH is required to increase TNF- $\alpha$ expression similar to accompanying bone loss (26). A previous study reported that despite severe hypogonadism, FSH stimulates TNF- $\alpha$ production, as demonstrated by loss-of-function studies indicating the presence of lowered TNF- $\alpha$ levels in FSH $\beta$-deficient mice (27). FSH triggers the production of TNF- $\alpha$ from macrophages and granulocytes in the bone marrow (27). In turn, TNF- $\alpha$ are able to stimulate osteoclastogenesis directly and strongly synergise with IL-1 and RANKL to promote osteoclast differentiation and activation (28). FSH may influence bone mineral density by modulating the activity of the osteoresorptive cytokine IL-1 $\beta$. Furthermore, FSH increases the secretion of IL-1 $\beta$, TNF- $\alpha$ and IL- 6 as surface expression of FSHR on monocytes increases (29).

FSH accelerated the progression of induced experimental periapical lesions, which may be mediated by increasing secretion of osteoresorptive cytokines (TNF- $\alpha$ and IL-1 $\beta$ ) and altered RANK/OPG ratios. The findings of the present study suggest that FSH, independent of oestrogen, may aggravate periapical bone loss by FSH receptors, which may serve an important role in the immune and inflammatory response of the host to root canal and periradicular infection during menopause.

\section{Acknowledgements}

The current study was supported by grants from the National Natural Science Foundation of China (grant no. 81120108010) and the Pre-National Basic Research Program of China (973 Plan; grant no. 2012CB722404).

\section{References}

1. Han NR, Kim HY, Yang WM, Jeong HJ and Kim HM: Glutamic acid ameliorates estrogen deficiency-induced menopausal-like symptoms in ovariectomized mice. Nutr Res 35: 774-783, 2015.

2. Wei S, Gong Z, An L, Zhang T, Dai H and Chen S: Cloprostenol and pregnant mare serum gonadotropin promote estrus synchronization, uterine development, and follicle-stimulating hormone receptor expression in mice. Genet Mol Res 14: 7184-7195, 2015

3. Santi D, Granata AR and Simoni M: FSH treatment of male idiopathic infertility improves pregnancy rate: A meta-analysis. Endocr Connect 4: R46-R58, 2015.

4. Herrera-Luna CV, Scarlet D, Walter I and Aurich C: Effect of stallion age on the expression of LH and FSH receptors and aromatase P450 in equine male reproductive tissues. Reprod Fertil Dev 6 Jul, 2015 (Epub ahead of print).

5. Cannon JG, Kraj B and Sloan G: Follicle-stimulating hormone promotes RANK expression on human monocytes. Cytokine 53: 141-144, 2011.

6. Robinson LJ, Tourkova I, Wang Y, Sharrow AC, Landau MS, Yaroslavskiy BB, Sun L, Zaidi M and Blair HC: FSH-receptor isoforms and FSH-dependent gene transcription in human monocytes and osteoclasts. Biochem Biophys Res Commun 394: 12-17, 2010.

7. Petrova NL, Petrov PK, Edmonds ME and Shanahan CM: Inhibition of TNF- $\alpha$ Reverses the pathological resorption pit profile of osteoclasts from patients with acute Charcot osteoarthropathy. J Diabetes Res 2015: 917945, 2015.

8. Tolias D, Koletsi K, Mamai-Homata E, Margaritis V and Kontakiotis E: Apical periodontitis in association with the quality of root fillings and coronal restorations: A 14-year investigation in young Greek adults. Oral Health Prev Dent 10: 297-303, 2012.

9. Zhang $X$ and Peng B: Immunolocalization of receptor activator of NF kappa B ligand in rat periapical lesions. J Endod 31: $574-577,2005$.
10. Xiong H, Peng B, Wei L, Zhang X and Wang L: Effect of an estrogen-deficient state and alendronate therapy on bone loss resulting from experimental periapical lesions in rats. J Endod 33: 1304-1308, 2007.

11. Zhang X, Peng B, Fan M, Bian Z and Chen Z: The effect of estrogen deficiency on receptor activator of nuclear factor kappa $\mathrm{B}$ ligand and osteoprotegerin synthesis in periapical lesions induced in rats. J Endod 33: 1053-1056, 2007.

12. Liu S, Cheng Y, Fan M, Chen D and Bian Z: FSH aggravates periodontitis-related bone loss in ovariectomized rats. J Dent Res 89: 366-371, 2010.

13. Suzuki N, Takimoto K and Kawashima N: Cathepsin K inhibitor regulates inflammation and bone destruction in experimentally induced rat periapical lesions. J Endod 41: 1474-1479, 2015.

14. Velickovic M, Pejnovic N, Mitrovic S, Radosavljevic G, Jovanovic I, Kanjevac T, Jovicic N and Lukic A: ST2 deletion increases inflammatory bone destruction in experimentally induced periapical lesions in mice. J Endod 41: 369-375, 2015.

15. Lin SK, Hong CY, Chang HH, Chiang CP, Chen CS, Jeng JH and Kuo MYP: Immunolocalization of macrophages and transforming growth factor- $\beta 1$ in induced rat periapical lesions. J Endod 26: 335-340, 2000.

16. Sun L, Peng Y, Sharrow AC, Iqbal J, Zhang Z, Papachristou DJ, Zaidi S, Zhu LL, Yaroslavskiy BB, Zhou H, et al: FSH directly regulates bone mass. Cell 125: 247-260, 2006.

17. Rawlinson SC, Boyde A, Davis GR, Howell PG, Hughes FJ and Kingsmill VJ: Ovariectomy vs. hypofunction: Their effects on rat mandibular bone. J Dent Res 88: 615-620, 2009.

18. Gomes-Filho JE, Wayama MT, Dornelles RC, Ervolino E, Yamanari GH, Lodi CS, Sivieri-Araújo G, Dezan-Júnior E and Cintra LT: Raloxifene modulates regulators of osteoclastogenesis and angiogenesis in an oestrogen deficiency periapical lesion model. Int Endod J 48: 1059-1068, 2015.

19. Pitari MR, Rossi M, Amodio N, Botta C, Morelli E, Federico C, Gullà A, Caracciolo D, Di Martino MT, Arbitrio M, et al: Inhibition of miR-21 restores RANKL/OPG ratio in multiple myeloma-derived bone marrow stromal cells and impairs the resorbing activity of mature osteoclasts. Oncotarget 6: 27343-27358, 2015.

20. Sağlam M, Köseoğlu S, Hatipoğlu M, Esen HH and Köksal E: Effect of sumac extract on serum oxidative status, RANKL/OPG system and alveolar bone loss in experimental periodontitis in rats. J Appl Oral Sci 23: 33-41, 2015.

21. Duan P, Wang ZM, Liu J, Wang LN, Yang Z and Tu P: Gene polymorphisms in RANKL/RANK/OPG pathway are associated with ages at menarche and natural menopause in Chinese women. BMC Womens Health 15: 32, 2015.

22. Duan P, Wang ZM, Liu J, Wang LN, Yang Z and Tu P: Association of gene polymorphisms in RANKL/RANK/OPG system with hypertension and blood pressure in Chinese women. J Hum Hypertens 29: 749-753, 2015.

23. Tu P, Duan P, Zhang RS, Xu DB, Wang Y, Wu HP, Liu YH and Si L: Polymorphisms in genes in the RANKL/RANK/OPG pathway are associated with bone mineral density at different skeletal sites in post-menopausal women. Osteoporos Int 26: 179-185, 2015.

24. Xu XJ, Shen L, Yang YP, Zhu R, Shuai B, Li CG and Wu MX: Serum $\beta$-Catenin levels associated with the ratio of RANKL/OPG in patients with postmenopausal osteoporosis. Int J Endocrinol 2013: 534352, 2013.

25. Marazzi J, Kleyer J, Paredes JM and Gertsch J: Endocannabinoid content in fetal bovine sera-unexpected effects on mononuclear cells and osteoclastogenesis. J Immunol Methods 373: 219-228, 2011.

26. Cenci S, Toraldo G, Weitzmann MN, Roggia C, Gao Y, Qian WP, Sierra O and Pacifici R: Estrogen deficiency induces bone loss by increasing $\mathrm{T}$ cell proliferation and lifespan through IFN-gamma-induced class II transactivator. Proc Natl Acad Sci USA 100: 10405-10410, 2003.

27. Karadag F, Ozcan H, Karul AB, Yilmaz M and Cildag O: Sex hormone alterations and systemic inflammation in chronic obstructive pulmonary disease. Int J Clin Pract 63: 275-281, 2009.

28. Kobayashi K, Takahashi N, Jimi E, Udagawa N, Takami M, Kotake S, Nakagawa N, Kinosaki M, Yamaguchi K, Shima N, et al: Tumor necrosis factor alpha stimulates osteoclast differentiation by a mechanism independent of the ODF/RANKL-RANK interaction. J Exp Med 191: 275-286, 2000.

29. Lam J, Takeshita S, Barker JE, Kanagawa O, Ross FP and Teitelbaum SL: TNF-alpha induces osteoclastogenesis by direct stimulation of macrophages exposed to permissive levels of RANK ligand. J Clin Invest 106: 1481-1488, 2000. 